\title{
MCQ Examinations for Large Number of Students: Design and Grading
}

\author{
Mohammed Ghazy ${ }^{1,2} \&$ Atef Ata ${ }^{2, *}$ \\ ${ }^{1}$ Dept. of Aerospace Engineering, KFUPM University, Dhahran 31261, Saudi Arabia \\ ${ }^{2}$ Dept. of Engineering Math \& Physics, Alexandria University, Alexandria 21544, Egypt \\ *Corresponding author: Dept. of Engineering Math. \& Physics, Alexandria University, \\ Alexandria 21544, Egypt. E-mail: atefa@alexu.edu.eg
}

Received: January 22, 2020 Accepted: February 27, 2020 Published: March 20, 2020

doi:10.5296/ije.v12i1.16319 URL: https://doi.org/10.5296/ije.v12i1.16319

\begin{abstract}
In this paper, a proposal for a criterion for designing and implementing MCQ exams in engineering mechanics course is introduced. The special case of a very large number of engineering students in Alexandria University is considered as a case study. In this case, a quick, though accurate and fair, method of evaluating students' performance is a must especially when using the credit hour system. Full Multiple-Choice Questions (MCQ) and computer based exams become so common. However, hybrid/subjective or total subjective exams are still in use in some institutions. Even though the process of implementing MCQ exams is well known and mature in humanity courses, in technical courses, sometimes, this process is not straightforward. It may depend on experience as it becomes well established and of minimum time after being applied for many years. In addition, the technical ability of the used software gives instructors more capabilities in analyzing results and establishing a basis for learning statistics. The case of coordinated engineering mechanics course with large number of students is emphasized. A hierarchy of procedures starting from ideas of questions till finishing grading and inputting grades in bulk into online system is formulated. The proposed methodology is of low cost and not so sensitive to technical abilities of software. Also, it doesn't require instructors with detailed computer knowledge. Finally, it can be used by instructors in other universities as it formulated to be of generic nature.
\end{abstract}

Keywords: learning analytics, MCQ exams, engineering mechanics, multiple models 


\section{Introduction}

The advantages and disadvantages of MCQ exams were discussed from the point of view of instructors and students by Ghazy \& Ata (2019). Similar to classical examinations, MCQ exams should measure all the learning outcomes of the course. Weimer (2018) indicated that this objective should be taken into consideration when designing MCQ exams. Computerbased exams can also help in measuring other sets of outcomes predetermined by the department and institution. Thus, MCQ exams are advantageous on the course, department, and institution level. In institutions with large number of students where the examination process requires more efforts and can't be managed totally by instructors, there can be a separate unit for grading exams. In such situation, computer based exams are the only exam formats that can be used. With the progress in online learning programs, and online bachelor degrees offered by some universities, using MCQ exams is essential where students from all over the world can't attend physically in-class exams (Weimer, 2018). The wide usage of online courses necessitates employing a type of questionnaire that can be implemented in computer based exams to allow immediate grading (Vulic et. al., 2017).

As engineering mechanics is a basic course in almost all engineering programs, different approaches were developed to improve many aspects related to its learning process (Persaud \& Smit, 2017; Zachary et. al., 2000). Recently, MCQ examinations are applied in engineering mechanics course in many universities all over the world, after being used extensively in humanity courses for years. Instructors of humanity courses used computer-based examinations and surveys to predict and improve students' performance as Ghazy (2017) did to measure student's expectations at the beginning of a course about oral presentation skills. However, when it comes to a technical engineering course such as engineering mechanics with different specific nature than humanity courses, some other parameters should be taken into consideration. The problems in the course need to be modeled and the equations of motion should be derived correctly. So the instructors are required to design their examinations carefully to evaluate the students and analyze the results correctly and efficiently. Definitely, there will be some disadvantages in addition to many advantages when applying MCQ exams (Ghazy \& Ata, 2019; Weimer, 2018). Advantages of MCQ exams when applying credit hour systems dominate. For instructors it enables them to grade the exam very fast and analyze the results in an easy way. When large number of students enrolled in the course, which is the case in some big universities, grading thousands of exams with classical format takes too much time and efforts of instructors. In such as a case the instructor with research duties finds difficulty achieving the institution key performance indicators in research.

One of the disadvantages of MCQ exams is that students can guess the correct answer without having to derive or calculate it. This is why some of them think that these exams are easier than classical ones. An instructor can partially solve this issue by changing the weights of the correct and incorrect choices. As there are fewer human factors affecting the grading process in MCQ exams, students usually trust the grades they get from these exams. However, in MCQ exams, many small problems can be included; some problems of descriptive nature like those in engineering mechanics cannot be included. Such problems need qualitative grading process. Drawing the free-body-diagram of a particle or a rigid-body is an example of these problems. 
However, there is still a chance to include these problems implicitly in MCQ exams. Students usually get some marks from these descriptive topics with qualitative way of grading even with partial correct answer. Actually, MCQ exams do not help in improving writing styles and other communication skills of engineering students. Instructor can use the large number of MCQ exams over years to figure out which part of the syllabus needs more efforts when explained to students.

In this paper, a criterion to apply MCQ exams in engineering mechanics course is introduced. In the second section, flow charts for creating multiple models are represented. In the third section, a flow chart of the grading process is presented. In the fourth section, a case study is analyzed and discussed. Finally in the last section conclusions are drawn.

\section{Creating Multiple Models}

Normally for large number of students there are many instructors teaching the course. The midterm and the final examinations should be unified for all students. To create multiple models examination, the suggestions from all instructors are collected and the first draft is formed. Each instructor should answer the draft and determine whether it is accurately designed in terms of time and difficulty. After this step, multiple models should be created and then reviewed for correct answers. Creating multiple models from the draft can be done using Microsoft Excel, Mathcad, or Matlab. After making sure that all answers are correct, the final multiple models could be printed. A flowchart for this process is shown in Figure (1).

\section{Grading the Examination Sheets}

After the examination, all answer sheet are scanned for grading using commercial software (Remark Office for example). The grading process consists of two main steps; the first one is to make sure that the software reads all the bullets correctly and it takes sometimes to clear all errors created by students such as double shading, blur shading, and blank answers. The second step is designing the answer scheme with weight for every answer. The easy way is the same weight for each answer but different weights are also available for the software. After grading is complete, many reports can be extracted from the software for analysis. To make sure everything is fine before announcing the final results, $10 \%$ of all the answer sheets should be graded manually. A flowchart for the grading process is shown in Figure (2). 


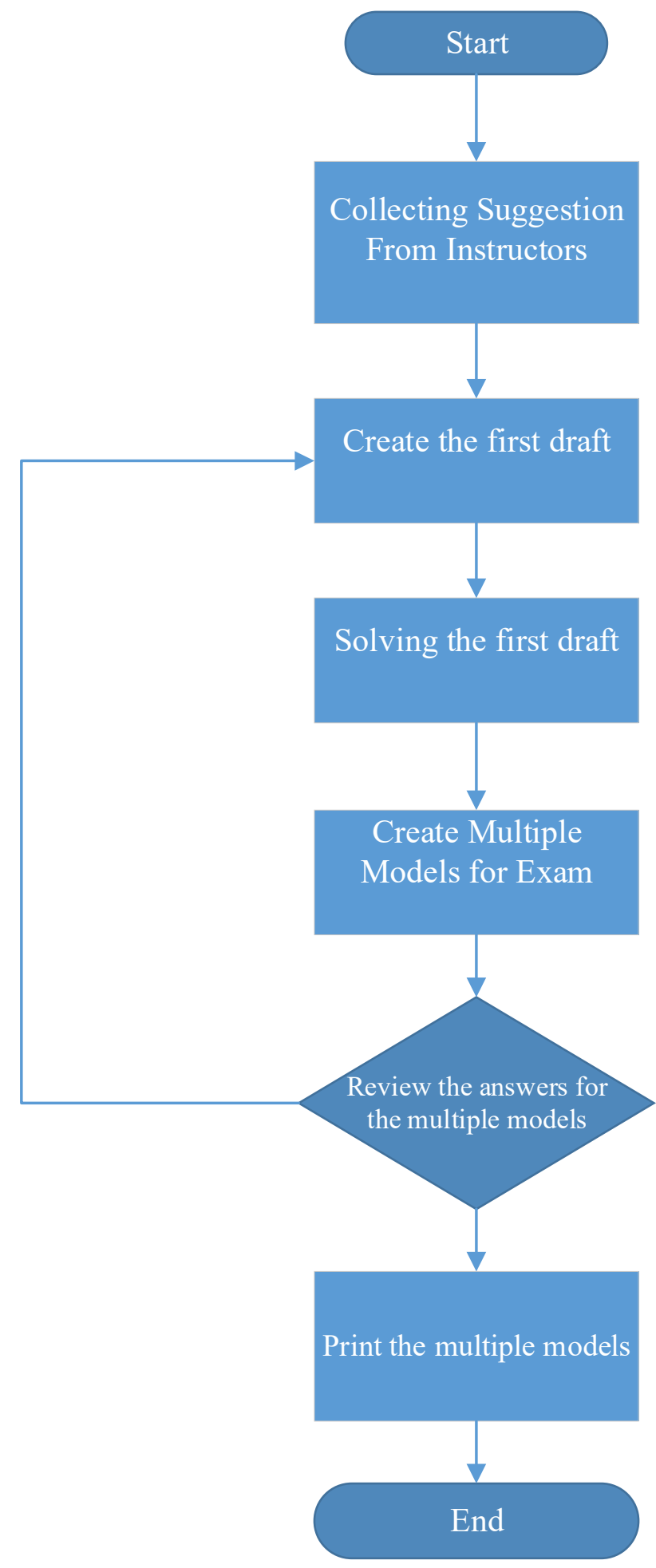

Figure 1. Flowchart for Creating Multiple Models Examination 


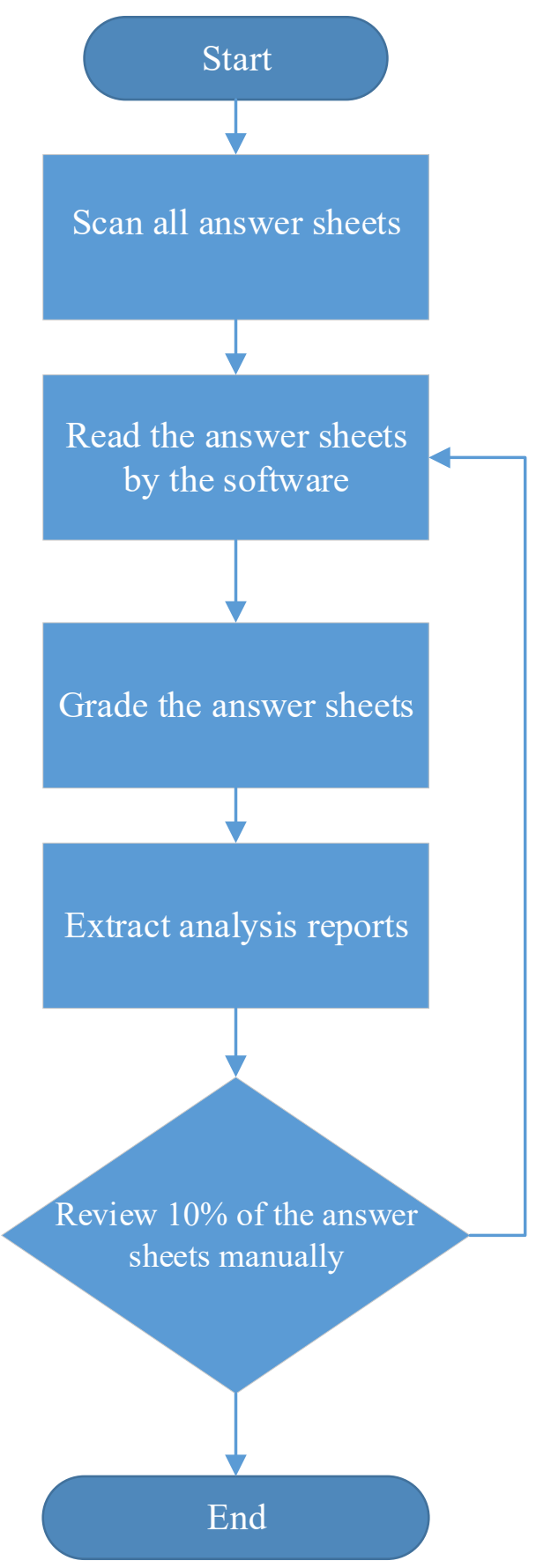

Figure 2. Flowchart for the Grading Process

\section{Case Study}

In this section a traditional question is compared with an MCQ exam question in engineering statics and engineering dynamics. The authors have a long experience in teaching and examining engineering mechanics for large number of students. Many forms of examinations were carried out and finally the MCQ form of examinations proves to be suitable for the situations where large number of students registered the course and the time slot for announcing 
the results is too narrow for the students to register for the second or summer semester.

The main advantage of classical written exam is the ability for the instructors to check how the students approach the problem and the way they utilize to search for the solution. It is also easier to examine the students in some aspects, which it will be implicitly included in MCQ examinations such as drawing the free-body-diagram in engineering mechanics problem. This free body diagram is very important in understanding the force analysis procedure and what will happen if the force is transferred along its line of action of a force parallel to itself in another point of the plane or space. It is also possible to examine the students in some analytical expressions and how they can integrate their knowledge in mathematics, calculus, and analytical geometry in deriving some expressions. The drawback of these kind of questions is that some students started the derivation correctly and they lost their way in the middle of the problem. They are trying to cheat the instructor by writing so many equations and expression and without reaching the final goal, they wrote it as if they derived it correctly. This put a burden on the shoulder of instructors to grade the students fairly and accurately and he has to follow up the answer and sometimes do it himself by hands to find out where the students cheated.

For a group of students up to 150 students, the efforts spent in designing MCQ examination with multiple models and the classical written examination with manual grading is almost the same. In MCQ examination, the efforts are mostly devoted to creating multiple models, solving all problems to make sure that all answers are correct. On the other hand, it is much easier to set classical essay examinations but more efforts are required in manual grading. As the number of students is increasing, the MCQ examination with multiple models is the best choice.

There are many applications in the market for MCQ grading and the famous software is Remark Office. This software is very effective in grading and analyzing the marks. All questions can be analyzed and valuable information can be deducted from the various analysis reports generated by the software. This enables the instructors to reassign the marks, redistribute the grades, or even cancel any questions without affecting the results. In addition, the instructors can change the weight of each question.

The main problem in evaluating the exam paper is the ability of the students to cheat especially in case of a single exam model for large number of students. It is highly recommended that the instructors use multiple examination models and the question paper should be connected to the answer sheet by a code or barcode or any hidden symbol. Also the exam paper should be stapled to the answer sheet to make sure that the students will answer only their questions and do not try to cheat. A simple number or barcode is printed in both papers.

Although the instructors apply all these measures, one can find that some students are trying to wipe out their exam code and try to mark their neighbors' code and get help instead of trying to solve. The percentage of students doing this fault is small and it could be known easily during scanning of the answer sheet or during grading. This is simply because many grading software are sensitive to any change in the resolution of the exam paper.

From the previous experience in teaching engineering mechanics course before 2009, we 
usually receive around 2500 every year in the Fall intake. At the end of each semester, these 2500 examination papers should be graded in a fair and accurate way. Normally for classic essay questions, the students should answer six questions in three hours exam. To make sure that each student got his fair marks, one question is graded by the same academic staff for all students and this process takes nearly 21-30 days between grading and reviewing the total amount of the answer sheets.

Since 2009, when we started applying the MCQ type examinations, we found how easy the grading is, and it saves more time and effort. In table (1) below, a list of needed time in hours for each step in grading 2500 sample with 30 questions and 5 choices for each question is presented. It should be noted that $10 \%$ of the whole sample should be graded manually to make sure that no errors appear in the results.

Table 1. Time Taken in Grading 2500 Answer Sheets

\begin{tabular}{ll}
\hline \multicolumn{1}{c}{ Step } & Time in Hours \\
\hline $\begin{array}{l}\text { Designing and solving the first model, designing multiple models, reviewing } \\
\text { multiple model }\end{array}$ & 48 hours \\
Scanning answer sheets & 3 hours \\
Designing the reading template & 0.5 hour \\
Reading the answer sheets & 1 hours \\
Reviewing and correcting the reading data & 1 hours \\
Designing the grading template & 0.25 hour \\
Grading the answer sheets and extracting the reports & 0.25 hour \\
Reviewing $10 \%$ of the answer sheets manually & 20 hours \\
Total elapsed time & 74 hours \\
\hline
\end{tabular}

In case of preparing the MCQ examinations from scratch and there is no question bank, it takes between 2-3 working days to be completed. This elapsed time can be reduced considerably when question banks are available. These days are not considered as a wasting time since the exam can be finalized during the normal semester activities. The most important part is grading and announcing the results after the examination so the students can register for the next semester. The total period will be only 3-4 days as a maximum compared to nearly one month for the essay question.

It should be mentioning that even before a simple check one can notice big differences among results. Some sections of students or some questions within the same model may show big difference in average grade. It will be obvious which sections or questions are different in average in the analysis reports. In this regard, the commercial grading software plays an important role in saving grading time. This ability puts certain level of confidence in the grading process. If an average of a model is largely different from the other models, then it might be re-graded if the problem is in the grading process or scanning the papers. If scanning 
and grading processes are correct, this model can be submitted back to instructor to check if there are typographical or technical errors. The decision, to solve this problem later, depends on the source of the problem.

\section{Conclusion}

MCQ exams are necessary for large number of students. The introduced criteria for creating and grading such exams in this paper can be used by instructors to save their times and obtain MCQ examinations of fast evaluation and accurate analysis. Analysis reports of the answer schemes can be used to derive the status of a student, whether he attended the lectures or practiced the course at tutorial or at home. Also, weaknesses of individual student or a group of students can be identified. For the number of students less than 150, the effort in essay examination and MCQ examination are almost the same. In this case, the essay examination is preferable since it enables the instructor to examine the students in descriptive topics. In addition, recorded results of large numbers of MCQ exams can be used in adaptive learning process.

\section{References}

Ghazy, M., \& Ata, A. (2019). Computer-Based Examinations in Engineering Mechanics Course; Necessity and Benefits. The First International Conference on Pedagogical Innovation and Technology Enhanced Learning (APITEL 2019), October 26-27, Alexandria, Egypt. CD-ROM ISSN 2784-2682, Online ISSN 2768-2682

Weimer, M. (2018). Multiple-Choice Tests: Revisiting the Pros and Cons. Retrieved 21 February, 2018 from https://www.facultyfocus.com/articles/educationalassessment/multiple-choice-tests-pros-cons/

Vulic, J. et al. (2017). Data Analytics Informing MOOC Continuous Improvement. 7th International Learning Analytics and Knowledge Conference. Simon Fraser University, Vancouver, Canada, 13-17 March, pp. 63-73. http://ceur-ws.org/Vol1967/FLMOOCS_Paper4.pdf

Persaud, S., \& Smit, M. (2017). A $21^{\text {st }}$ Century Approach to Engineering Mechanics Education. International Conference on Engineering and Product Design Education, $7 \& 8$ September, Oslo and Akershus University, Norway. PP. 704-79. ISBN: 978-1-904670-84-1

Zachary, L.W., Sharp, J., \& Adams, B. M. (2000). Engineering Connections: Teaching Engineering Mechanics to K 12 Teachers.

Ghazy, M. (2017). Improving Communication and Presentation Skills in English for Engineering Students for Sustainability. International Journal of Quality in Education, 1, $1-12$. 


\section{Copyright Disclaimer}

Copyright for this article is retained by the author(s), with first publication rights granted to the journal.

This is an open-access article distributed under the terms and conditions of the Creative Commons Attribution license (http://creativecommons.org/licenses/by/3.0/). 